\title{
Optimized Feature Extraction for Temperature-Modulated Gas Sensors
}

\author{
Alexander Vergara, ${ }^{1,2}$ Eugenio Martinelli, ${ }^{3}$ Eduard Llobet, ${ }^{2}$ \\ Arnaldo D'Amico, ${ }^{3}$ and Corrado Di Natale ${ }^{3}$ \\ ${ }^{1}$ Institute for Nonlinear Science, University of California, San Diego, La Jolla, CA 92093-0402, USA \\ ${ }^{2}$ MINOS, Universitat Rovira i Virgili, Avda. Països Catalans, 26, 43007 Tarragona, Spain \\ ${ }^{3}$ Department of Electronic Engineering, University of Rome Tor Vergata, via del Politecnico 1, 00133 Roma, Italy
}

Correspondence should be addressed to Corrado Di Natale, dinatale@uniroma2.it

Received 26 January 2009; Accepted 3 April 2009

Recommended by Michele Penza

\begin{abstract}
One of the most serious limitations to the practical utilization of solid-state gas sensors is the drift of their signal. Even if drift is rooted in the chemical and physical processes occurring in the sensor, improved signal processing is generally considered as a methodology to increase sensors stability. Several studies evidenced the augmented stability of time variable signals elicited by the modulation of either the gas concentration or the operating temperature. Furthermore, when time-variable signals are used, the extraction of features can be accomplished in shorter time with respect to the time necessary to calculate the usual features defined in steady-state conditions. In this paper, we discuss the stability properties of distinct dynamic features using an array of metal oxide semiconductors gas sensors whose working temperature is modulated with optimized multisinusoidal signals. Experiments were aimed at measuring the dispersion of sensors features in repeated sequences of a limited number of experimental conditions. Results evidenced that the features extracted during the temperature modulation reduce the multidimensional data dispersion among repeated measurements. In particular, the Energy Signal Vector provided an almost constant classification rate along the time with respect to the temperature modulation.
\end{abstract}

Copyright (C) 2009 Alexander Vergara et al. This is an open access article distributed under the Creative Commons Attribution License, which permits unrestricted use, distribution, and reproduction in any medium, provided the original work is properly cited.

\section{Introduction}

In a seminal paper by Zaromb and Stetter [1] the possibility to approach the quantitative and the qualitative analysis of simple and complex gas mixtures with solid-state sensor arrays and intelligent signal processing algorithms was introduced. Since that, countless examples of this approach appeared in literature and, among the used sensors technologies, metal-oxide semiconductor gas sensors were largely investigated [2-5]. Even if these researches corroborated the initial hypothesis, the practical application of solid-state sensors is still limited in practice by nontolerable instability of sensors signals. This is manifested as a nonpredictable temporal variation of the sensor signal whose more evident consequence is the spread of sensors responses when the sensor undergoes the same gas exposure. Consequently, calibrated sensor response patterns become, even at short time scale, obsolete providing false evaluations and requiring, as countermeasure, frequent calibrations. The instability of sensor signals derives from two main sources. The first is clearly found in the nature of the chemical and physical processes occurring in the sensor [6], such as ageing (e.g., the reorganization of the sensor surface) and poisoning (e.g., irreversible analyte binding). The second source of instability, often neglected, pertains to uncontrolled changes of the measurement conditions due for instance to fluctuations of the sample flow rate or the temperature of gas and sensors. In addition, other issues related to the experimental setup can induce either memory effects (such as systematic errors due to repeated measurement sequences) or short-term effects (system warm-up, thermal trends). These effects may also trigger the degradation of samples that in some cases can give rise to nonreproducible sensor responses that can be attributed to sensors drift. The sources of sensor instability 
due to nonaccurate measurement procedure can hopefully be counteracted by careful experimental designs.

The removal of those effects of physical and chemical origins resulting in sensors instability is a complicated task that requires a deep knowledge of the physics and the chemistry involved in the sensing process and, even more complicated, in the control of those technological steps related to the sensor fabrication process. Nonetheless, disregarding the "physical" approach to sensor stability, several authors demonstrated that it is possible to improve the sensor performance by an intelligent selection of the sensor features [7], by an optimization of the sensor operating conditions [8], and by a clever design of signal processing algorithms [9].

Feature extraction is defined as the operation necessary to extract from the temporal evolution of the sensor signal a set of synthetic descriptors that can be used in computations aimed at identifying the gas components in a mixture and their concentration. Features are practically related to the measure of the sensor signal in two conditions defined by the absence and the presence of the sample. The signal measured in these two states can be combined in order to obtain different quantities. For instance, in case of chemically sensitive resistances the simplest features that can be defined are, $R_{\text {gas }}-R_{\text {ref }}$, and $\left(R_{\text {gas }}-R_{\text {ref }}\right) / R_{\text {ref }}$ where $R_{\text {gas }}$ and $R_{\text {ref }}$ are the sensor resistance measured in presence of the sample and the reference atmosphere, respectively. It is known that even these simple quantities have different stability properties [10]. A methodology suggested to improve the sensor performance consists in the time modulation of the sensor signal obtained changing at least one of the sensor operating conditions. In case of metal-oxide semiconductor gas sensors, since the sensor properties are strongly dependant on the sensor temperature [11], the modulation of temperature is an easy method to obtain a time variable sensor signal. In these sensors temperature modulation is expected to alter the kinetics of both adsorption and reaction processes taking place at the sensor surface. Temperature modulation leads to the development of response patterns, which may be characteristic of the species being detected. In other words, by retrieving information from response dynamics, new response features are obtained that can confer more selectivity to metal oxide sensors. Indeed, several authors have developed this strategy and applied several techniques to extract features that are important for the discrimination or quantification of gases. Fast Fourier Transform (FFT) and Discrete Wavelet Transform (DWT) have been used as feature extraction tools in several studies [12-17]. Various researches shown that the features extracted from temperature modulated gas sensors are more stable with respect to sensors operated at fixed temperature [1822]. An important set of parameters to be adjusted is defining the temperature modulation profile. In case of simple sinusoidal modulation some results evidenced the existence of optimal modulation frequencies whose application results in a general improvement of selectivity and stability $[14,15]$.

Concerning the expected efficiency of the methods based on optimized features extraction, signal processing, and operative conditions it is important to consider that it is rather unlikely that these approaches can remove the degradation of physical and chemical properties of a sensor, but since the instability is also due to the fluctuations of the experimental conditions signal processing optimization can be considered as the search of features that are less sensitive to those quantities that fluctuate among repeated measurements.

In this paper, we illustrate the stability properties of a number of distinct features derived from systems theory, a discipline defining the instruments to study and represent complex time evolving systems.

In this study, the features are simultaneously extracted from a temperature-modulated array of metal-oxide semiconductor gas sensor. The stability is evaluated in a fourclasses pattern recognition problem assuming that improved stability results in a better classification of the four classes. Sensors were operated in a discontinuous mode temperature modulation where they are normally kept nonpowered and the temperature modulation is applied only during the time interval when feature extraction is performed [23].

\section{System Theory and Sensors Features Extraction}

Elements of systems theory have been used in the past to model sensors behavior considering not only the steady state changes but also the dynamic evolution of signals. Attempts to model these behaviors using either time series or analytical approximation have been shown in the past [24-27], and the use of specific methods to extract only the dynamic information was also demonstrated $[28,29]$. Nonetheless, only recently one of the central concepts for the analysis of dynamics systems, the phase space, has been taken into consideration. In order to define the phase space let us consider a system whose state is completely described by $n$ scalar variables. Different states corresponds to different points in an $n$-dimension vector space defined by an orthonormal basis where each direction corresponds to one of the scalar variables. The main property of such space is the unique correspondence between points in the space and states of the system.

Practically, the information gained about a system, such as a sensor, is contained in the temporal evolution of the measured signal. Nonetheless, from the time dependence of this scalar quantity a number of novel variables can be generated. For instance, in mechanical systems, the observation of the position with the time gives place to variables such as velocity (first derivative) and acceleration (second derivative) from which the phase space is constructed.

As a consequence, a system, observed through a single scalar quantity for a period of time, is represented by a sequence of points in the phase space. Once suitably parameterized with the time, this sequence forms a trajectory containing all the captured information about the phenomena underlying the system evolution. The relation between the observed signals and the internal variables forming the phase space has been demonstrated by the embedding theorem of Takens [30]. According to this theorem, the 


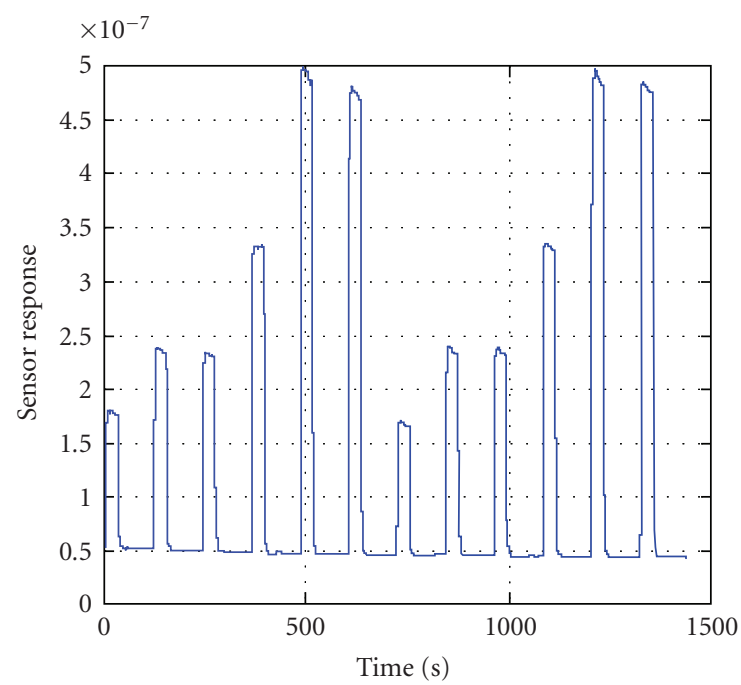

(a)

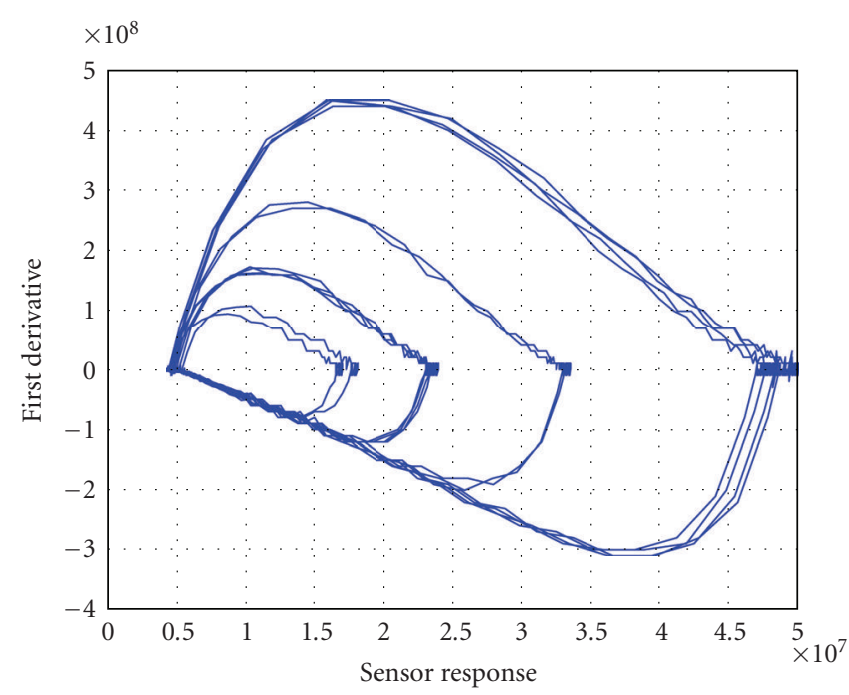

(b)

FIGURE 1: Responses of a $\mathrm{SnO}_{2}$ gas sensor in presence of different gas concentrations; (a) the sensor response in the time domain; (b) phase space of $\mathrm{SnO}_{2}$ sensor with $f(t)$ and $f^{\prime}(t)$ as canonical variables.

variables of the phase space are given by a number of differentiable transformations of the observed signal such as a set of independent coordinates $x(t), x\left(t+\tau_{1}\right), x(t+$ $\left.\tau_{2}\right), \cdots, x\left(t+\tau_{n}\right)$. Each variable of this set represents the experimental time series at different time samples (after a specific time delay $\tau_{n}$, a multiple of the sampling time step $\mathrm{T}_{\mathrm{s}}$ ). Any dynamic state of the system is therefore represented in a multidimensional phase space as $y(t)=$ $\left[x(t), x\left(t+\tau_{1}\right), x\left(t+\tau_{2}\right), \cdots, x\left(t+\tau_{n}\right)\right]$. The number of coordinates defining the phase space and the values of the time delays $\tau_{n}$ must be chosen suitably in order to obtain an optimal description of the phenomena. There are sufficient conditions to avoid false projection and ambiguities of the trajectories.

From a general point of view, a chemical sensor can be considered as a dynamic system whose response signal temporally evolves following, with its proper dynamics, the concentration of the analytes. The information is in the temporal evolution of a single scalar quantity (i.e., in the sensor signal) and from this time dependence, a number of new variables can be generated. The minimum set of variables able to describe the state and the evolution of the sensor comprises the response signal (i.e., the observed variable) and its first derivative. Therefore, a phase space can be defined by the orthonormal basis formed by the observed signal and its first derivative. The use of the phase space in the field of gas sensors was first introduced in 2003 [31]. In the Figure 1 the responses of $\mathrm{SnO}_{2}$ sensor in presence of different gas concentrations and the relative trajectories spanned in the phase space are shown.

The description of the trajectory in the phase space give rise to novel features related to the underlying phenomena that can be used to build more accurate gas identification models. The area spanned by the trajectory when it undergoes a transformation (e.g., during the adsorption) is a rather straightforward descriptor to quantify the process. In this quantity both the magnitude and the velocity of the sensor response are considered. However, the area is a descriptor that overlooks some characteristics of the trajectory evolution (i.e., the shape of the trajectory). This is the reason to include other descriptors, for example, the dynamic moments (DMs), that can describe also the morphology of the trajectories [32].

Among the possible quantities that can bring such notions, the following DMs are here discussed, all of them are defined as combination of the phase space variables:

$$
\begin{gathered}
\mathrm{DM} 2=\frac{1}{n} \sum_{i=0}^{n} x_{i} y_{i} \\
\mathrm{DM} 3_{P B}=\frac{\sqrt{2}}{2 n} \sum_{i=0}^{n}\left(x_{i}^{2} y_{i}-x_{i} y_{i}^{2}\right) \\
\mathrm{DM}_{S B}=\frac{\sqrt{2}}{2 n} \sum_{i=0}^{n}\left[2 x_{i}^{3}+3\left(x_{i}^{2} y_{i}-x_{i} y_{i}^{2}\right)\right] \\
\mathrm{DM} 3_{X}=\frac{1}{2 n} \sum_{i=0}^{n}\left(x_{i}^{3}-3 x_{i} y_{i}^{2}\right) \\
\mathrm{DM}_{Y}=\frac{1}{2 n} \sum_{i=0}^{n}\left(x_{i}^{3}-3 x_{i}^{2} y_{i}\right)
\end{gathered}
$$

where $\left.x(t)\right|_{t_{i}} ^{t_{i}+k}$ and $\left.y(t)\right|_{t_{i}+\tau} ^{t_{i}+k+\tau}=\left.x(t+\tau)\right|_{t_{i}+\tau} ^{t_{i}+k+\tau}$ are portions of the sensor signal $\left(t_{\mathrm{i}}=0,1,2,3, \ldots ; k=1,2\right.$, 3and $5 \mathrm{~s} ; \tau=1,2$ and $3 \mathrm{~s}$ ). Each DM describes a different morphological feature of the trajectory, so that the collective use of more than one moment is usually required for an exhaustive description of a phase space trajectory. The first gas sensor signal described by DMs was the quartz 


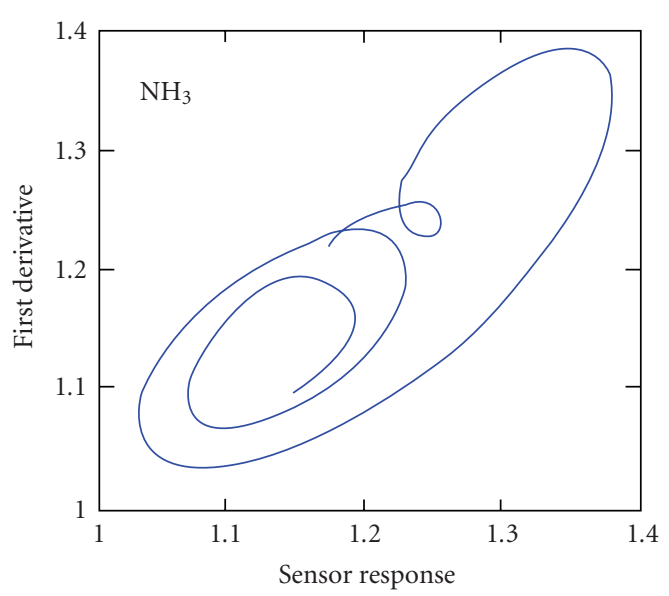

(a)

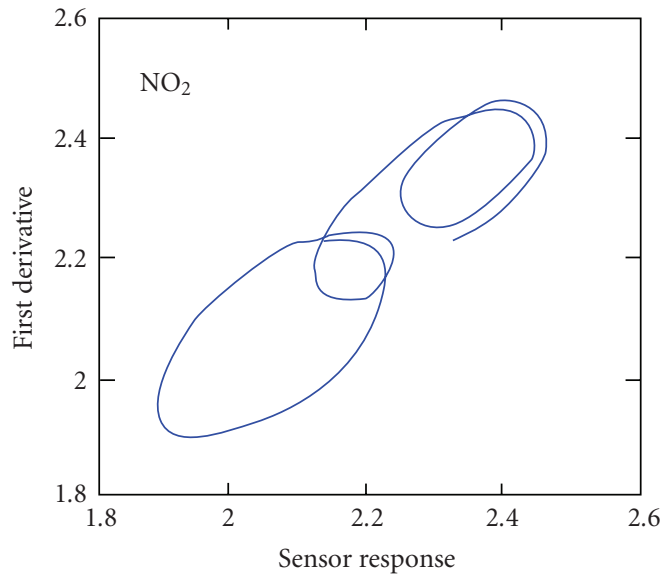

(b)

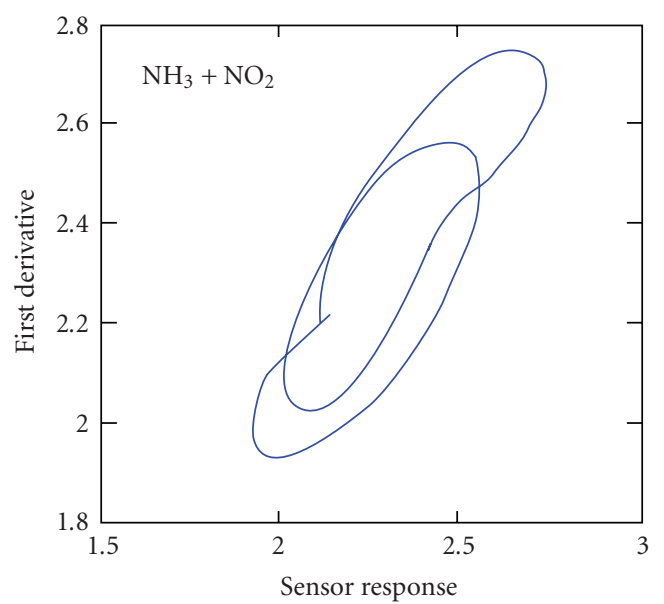

(c)

Figure 2: Example of the signal response of a tungsten oxide microhotplate sensor to ammonia (500 ppm), nitrogen dioxide (1 ppm) and ammonia + nitrogen dioxide $(500+1 \mathrm{ppm})$ in the phase space domain. The first derivative of the sensor response for a time lag equal to $3 \mathrm{~ms}$ ( $y$-axis) is plotted verses the sensor response ( $x$-axis). Reproduced from [33] Elsevier Science, with permission.

microbalance [33], whereas in the example here illustrated they are applied to temperature modulated metal-oxide gas sensors. In Figure 2 typical trajectories in the phase space of a tungsten oxide gas sensor in the presence of the gases are shown. The exposure to different gases and mixtures results in evidently distinct trajectories. Thes differences are transformed in quantities by the application of the dynamic moment.

Phase space is an example of a principle of the classical signal theory applied to the study of the time evolution of a gas sensor signal. Following the suggestions of signal theory let us consider a gas sensor array as a mathematical object, where the observable signals of each sensor are part of a multidimensional array. When the sensors simultaneously interact with the same chemical compounds, the signals of the sensors becomes characterized by a certain degree of mutual correlation. The amount of correlation may change according to the quality and quantity of the gas species to which the array is exposed. One of the quantities that can express the relationship between the sensors of an array is the mutual energy. Given two generic signals $x(t)$ and $y(t)$, the mutual energy in the interval $\left(t_{0}, t_{1}\right)$ is given by the following definition:

$$
\xi_{x y}=\int_{t_{0}}^{t_{1}} x(t) y(t) d t .
$$

The value of the energy likely depends on the affinitie between the gas species present and the sensor active layer. Hereafter, we call energy vector (EV) the following quantity:

$$
\begin{aligned}
\mathrm{EV}=\mid & \left|\begin{array}{llllllllll}
\xi_{1,1} & \xi_{1,2} & \ldots & \xi_{1, k} & \xi_{2,1} & \xi_{2,2} & \ldots & \xi_{2, k} & \ldots & \xi_{k-1, k-1} \\
& \ldots & \xi_{k, k}
\end{array}\right|
\end{aligned}
$$

where $k$ is the number of sensors in the array, and the generic vector element $\xi_{x y}$ is defined by (7). This method 


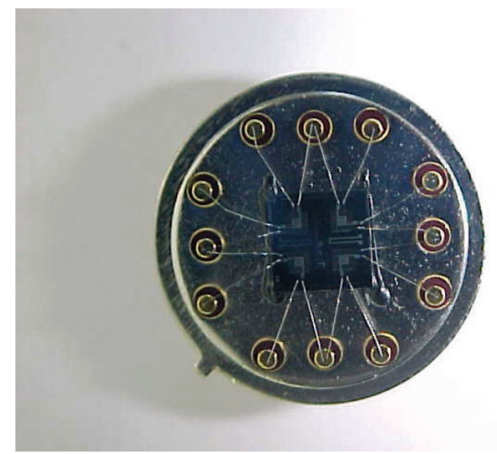

FIGURE 3: Four-element micro-hotplate array mounted on a standard TO-8 support.

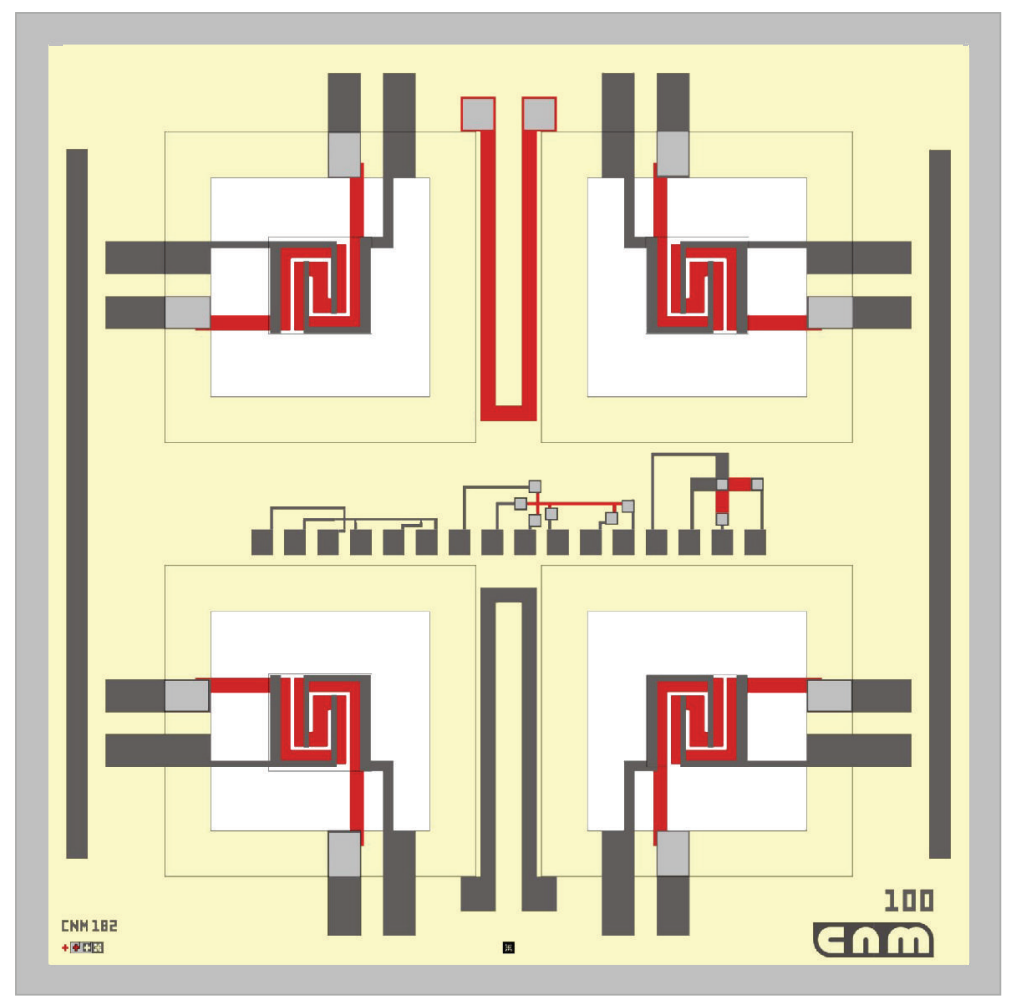

FIGURE 4: Chip layout where heating meanders and interdigited electrodes can be identified.

was originally proposed by Martinelli [34] and later more extensively applied to gas sensors arrays by Vergara et al. [35].

\section{Experimental}

3.1. Sensor Fabrication. Sensing layers were deposited over an integrated four microhotplates fabricated using microsystems technology. Basically, each sensor was formed by a gas-sensitive layer, interdigitated electrodes, insulating layers and a poly-silicon heater, all stacked on a $0.2 \mu \mathrm{m}$ thick $\mathrm{Si}_{3} \mathrm{~N}_{4}$ membrane grown by low-pressure chemical vapor deposition (LPCVD). The micro hotplate was characterized by a temperature coefficient of resistivity (TCR) equal to $6.79 \times 10^{-4}$. Heaters resistance was also used to measure the actual temperature of the sensor membranes. The power consumption was about $80 \mathrm{~mW}$ when working at $480^{\circ} \mathrm{C}$ and the thermal response of the coated membranes was measured to be near 20 milliseconds (thermal response was estimated to be about 10 milliseconds in bare devices). The technological fabrication steps of the substrates are reported elsewhere [36, 37]. Finally, the 4-element microhotplate array was packaged on a standard TO-8 package (see Figures 3 and 4). Each chip had 4 membranes, that can be functionalized with proper sensing layers, the size of which was $450 \times 450 \mu \mathrm{m}^{2}$ and the electrode area was $50 \times 50 \mu \mathrm{m}^{2}$.

The sensor array was formed by four thick-film metal oxide layers. The following materials were used: $1 \% \mathrm{Pt}$-doped and $1 \%$ Pd-doped tin oxide (Sensors A and B) and 1\% Ptdoped and 1\% Pd-doped tungsten oxide (Sensors C and D). These were deposited using a drop coating technique. A paste 
was prepared dispersing the active material $\left(\mathrm{SnO}_{2}\right.$ or $\mathrm{WO}_{3}$ nanopowders $+1 \% \mathrm{Pd}$ or $\mathrm{Pt}$ ) in glycerol. This process was repeated four times in order to obtain four different layers on the different membranes that integrated a micro-sensor array. The as-deposited films were dried in an oven at $180^{\circ} \mathrm{C}$ for 2 hours. Subsequently; they were annealed in situ at $480^{\circ} \mathrm{C}$ for 2 hours. The purpose of annealing was to ensure a good adherence of the active material to the sensor substrates and to burn out the organic vehicle (i.e., glycerol) used in the paste. The selection of these materials was based on previous experience for an analogous gas analysis application [38].

3.2. Experimental-Setup. An array composed of seven microhotplate gas-sensors was placed in a $20 \mathrm{~mL}$ volume air-tight test chamber to measure vapors of acetaldehyde, ethylene, ethanol and ammonia. This chamber was connected to a continuous flow system that allowed us to obtain, with high reproducibility, different concentrations of pollutant gases diluted in dry air. Mass flow controllers provided the dilution of test gases (at the desired concentrations) in dry air. The total gas flow was set to $200 \mathrm{~mL} / \mathrm{min}$ and kept constant. The moisture level was kept to $10 \%$ of relative humidity (measured at $30^{\circ} \mathrm{C} \pm 1^{\circ} \mathrm{C}$ ) during all the experiments.

The signals of the micro-gas sensor array were measured when the operating temperature of sensors was modulated using an optimized multisinusoidal signal. The multisinusoidal signal was generated using a written-in-house MATLAB code. A set of six modulating frequencies (optimal frequencies selected in a systematic way) was employed to synthesize the temperature-modulating multisinusoidal signal. For the sake of simplicity, the same multisinusoidal signal was applied to the heating element of every sensor within the array. The frequencies composing this signal were $12.8 \mathrm{mHz}, 25.6 \mathrm{mHz}, 38.5 \mathrm{mHz}, 92.9 \mathrm{mHz}, 339.7 \mathrm{mHz}$ and $682.7 \mathrm{mHz}$. The first three frequencies are known to be more important for gas identification, while the last three frequencies are helpful both for identifying and quantifying the gases considered [39]. The range of modulating frequencies is far below the cut-off frequency of the metal oxide coated membranes, which is the inverse of their thermal response (i.e., $\left.(1 / 20) 10^{-3} s^{-1}=50 \mathrm{~Hz}\right)[22]$.

3.3. Databases. The following compounds were tested: acetaldehyde $(50 \mathrm{ppm})$, ethylene $(50 \mathrm{ppm})$, ethanol $(25 \mathrm{ppm})$, and ammonia $(50 \mathrm{ppm})$. Five sessions of measurements were performed. Each session consisted in 12 replicas of each exposure, resulting in a total of 240 measures; samples sequence, in each session, was randomized. Among the measurement sessions, sensors were powered off. Each measurement took 312 seconds corresponding to the time of application of the multisinusoidal temperature modulation. The whole experiment was performed in four months. During the entire measurement process, before and after each measurement, both sensors and their heating elements were not powered. Finally, the sensors were powered when the analyte was already into the sensors cell.

The sampling rate of the sensor signals was set at 100 samples per second, which was far higher than the frequency range applied to the temperature modulating signal. The peak to peak amplitude of the multisinusoidal current signal applied to the heater was equal to $2 \mathrm{~mA}$. It corresponds to a sensor operating temperature range between $220^{\circ} \mathrm{C}$ and $450^{\circ} \mathrm{C}$. Figure 5 shows a typical transient response from a $\mathrm{Pd}$-doped $\mathrm{SnO}_{2}$ gas sensor in the presence of $50 \mathrm{ppm}$ of ammonia when the measurement time was set to $312 \mathrm{~s}$.

3.4. Data Analysis. From the recorded signals the previously defined features called dynamic moments and energy vectors were calculated. For sake of comparison the following standard quantities were also calculated and considered in the analysis:

Steady state feature extraction $\Delta R / R_{0}$, where the $\Delta R$ is the shift of the resistance between the instant value of the measure and the initial baseline $\left(R_{0}\right)$ according to this formula:

$$
\begin{gathered}
\frac{\Delta R\left(t_{i}\right)}{R_{0}}=\frac{\overline{R\left(t_{i}\right)}-R_{0}}{R_{0}} \\
\overline{R\left(t_{i}\right)}=\frac{1}{5} \sum_{j=1}^{5} R\left(t_{i-j}\right)
\end{gathered}
$$

with $t_{\mathrm{i}}=0,1,2,3, \ldots$

(i) The integral of the sensor signal $\operatorname{INT}\left(x\left(t_{i}\right)\right)_{k}=$ $\int_{t_{i}}^{t_{i}+k} x(t) d t$ with $k$ values considered in the data analysis are $1,2,3$ and $5\left(t_{\mathrm{i}}=0,1,2,3, \ldots\right)$.

(ii) The Fast Fourier Transform (FFT) coefficients of the sensor signal calculated on the entire temperature modulation applied to the sensor [40];

In order to reduce the measurement time all the features, except FFT, have been calculated in the first 20 seconds of each measurement.

Partial Least Square Discriminant Analysis (PLS-DA) has been utilized as classifier to compare the performance of features. For validation purposes, the entire dataset was split in training and testing sub-dataset (144 and 96 measurements, resp.). The number of latent variables used for PLS-DA models [41] was optimized minimizing the generalization errors applying the Leave-One-Out Crossvalidation (LOO-CV).

\section{Results and Discussion}

Collected data was analyzed in order to study the performance of the defined features in a shorter time with respect to the whole time during which the temperature modulations was applied. To this scope, only the first twenty seconds of the sensors response were considered. Since FFT requires the entire modulation period to be efficiently calculated it was not included in this analysis. Features were calculated every second in the first $20 \mathrm{~s}$ considering sensor signal lengths of $1 \mathrm{~s}, 2 \mathrm{~s}, 3 \mathrm{~s}$, and $5 \mathrm{~s}$.

The classification rates are summarized in Figure 6. The results obtained in validation and calculated considering a signal portion of $1 \mathrm{~s}$ are shown. All the features shown in 


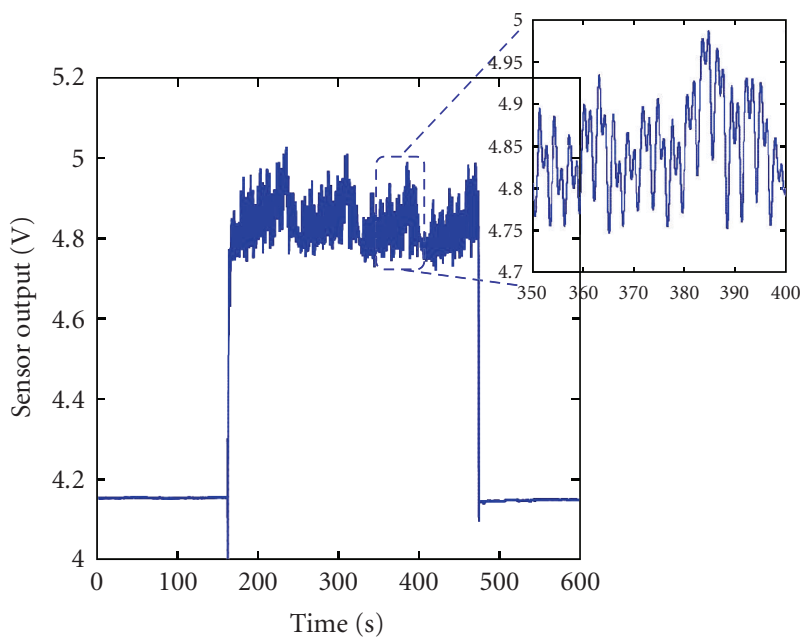

FIgURE 5: Typical time behavior of a gas sensor signal during the measurement phase.

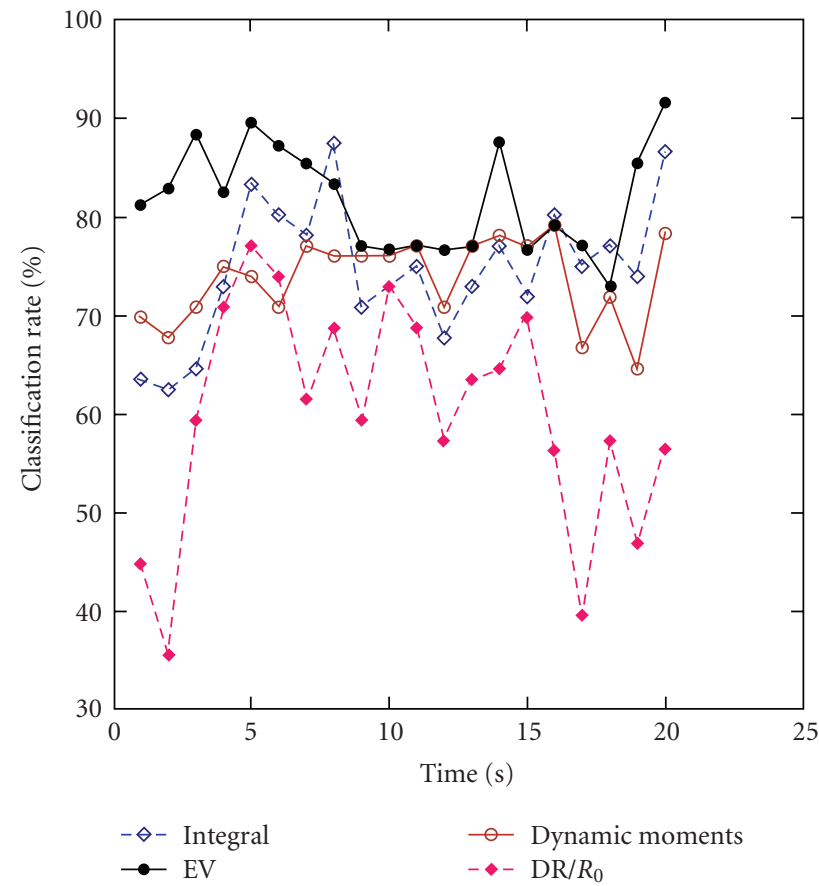

FIgURE 6: Classification rates in the validation phase using four features calculated with signals in the first twenty seconds of the temperature modulation. All features were calculated in a time window of $1 \mathrm{~s}$.

Figure 6 achieved more than $93 \%$ of correct classification in training. Among these features, EV achieved the highest classification rate equal to $91 \%$. Furthermore, EV provides discrimination rate higher than $80 \%$ in the first second of measurement the classification rate reaches $90 \%$ after $5 \mathrm{~s}$. Common features, such as $\Delta R / R_{0}$, give the worst performance in almost all the twenty seconds. Finally, it is important to note that the classification rates of EV and DMs are quite constant over the entire measurement time. EV results are even promising for real applications, in particular considering that a short measurement time results in reduced sensor power consumption. It is important to remark that the application of dynamic feature extraction contributes to a reduction of the measurement time as it was observed since several years ago applying a multiexponential fit to the signal of $\mathrm{SnO}_{2}$ based gas sensors [42].

The performance of features such as EV, Integral and DMs are clearly dependent on the signal behavior and their results could change considering different time windows. This effect is shown in Figure 7 where a summary of the classification rate of the above features considering a time window of $2 \mathrm{~s}$ is shown using a box-whisker plot. 


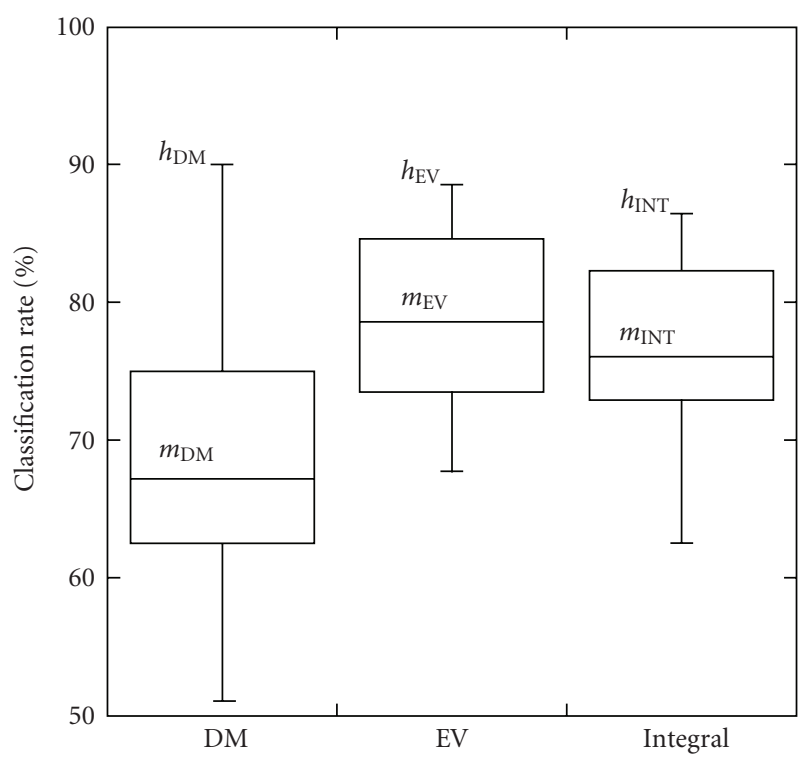

Figure 7: Box-plots of the classification rates in validation of Integral, Dynamic Moments and Energy Vector. Each features was calculated in a time window of 2 seconds.

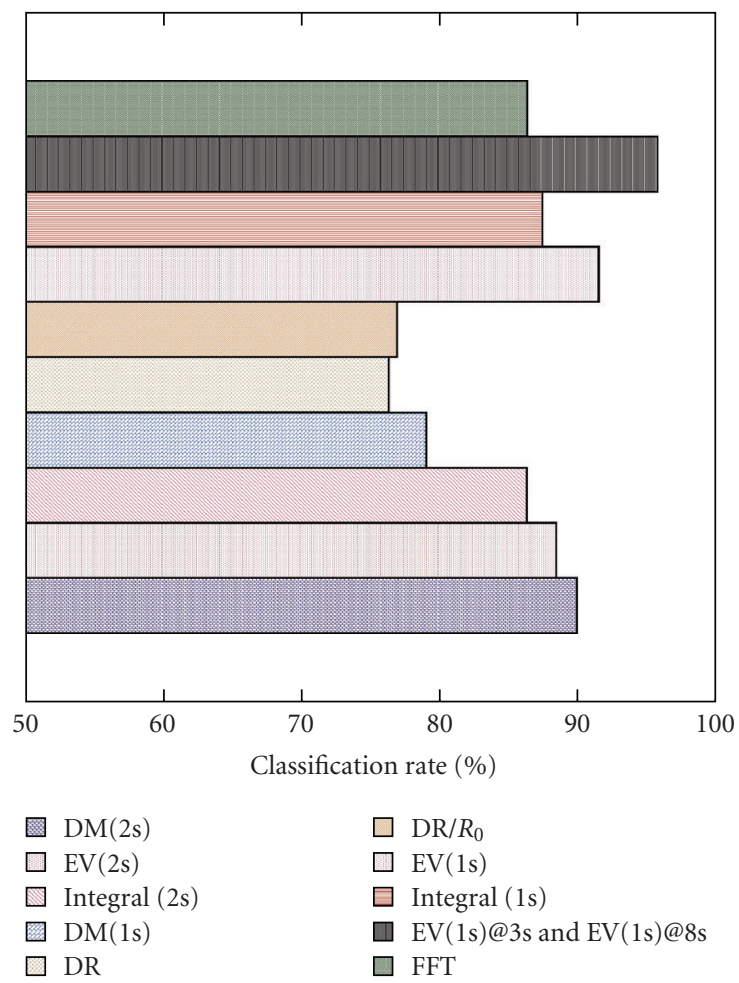

FIGURE 8: Comparison of the best classification rates in validation obtained by each considered feature.

DMs' have the highest classification rate $\left(\mathrm{h}_{\mathrm{MD}}\right.$, about $90 \%)$, but this occurs only in a particular situation determined by a particular matching of the signal with the requirements of DM. The box-plot for these descriptors is characterized by a low mean value $\left(\mathrm{m}_{\mathrm{MD}}\right)$ with respect to the $\mathrm{h}_{M D}$ confirming a large variability in the first $20 \mathrm{~s}$. A different behavior is observed for $\mathrm{EV}$, where the distribution of the classification rate is less dispersed than that of DMs. Finally, the integral shows the same order of variability of the EV but, generally, a lower classification rate. A supplementary investigation has been done considering the performance of the features calculated using $3 \mathrm{~s}$ and $5 \mathrm{~s}$ of the sensors signal portion. In this case, the classification rate was lower than the previous cases. 
A further improvement was obtained considering more EVs calculated at different times but using for each calculus a time lengths of $1 \mathrm{~s}$. EVs were calculated at $3 \mathrm{~s}$ and $8 \mathrm{~s}$. In this case the classification rate of validation reached $96 \%$ and almost $100 \%$ in training. In Figure 8 a summary of the best performance obtained in validation for each feature is shown.

The best result is obtained using a combination of two different EVs. This means that EVs did not provided totally overlapped information. These results are better appreciated considering that FFT, calculated with the whole experiment time of $312 \mathrm{~s}$, provided the $86 \%$ of correct classifications.

Concerning the performance of DMs, results show that it is possible to increase the classification rate but only in a precise interval of the signal evolution and combining different DMs. This last detail reinforces the efficiency of the recognition power of EV that was almost independent from the temperature modulation applied and, on the other hand, the difficulties to determine an optimized configuration for DMs features.

EV promising results are fundamentally due to the combination in these features of the relations between couples of sensor signals putting in evidence the crosscorrelation among sensors, and that this quantity is expected to be weakly sensitive to any instability source.

\section{Conclusions}

The stability of different kinds of features has been investigated utilizing an array of optimized multisinusoidal signal temperature modulated metal oxide gas sensors. For this measurement setup, results have evidenced that the dynamic features achieve better performance with respect to the standard feature extraction such as $\Delta R / R_{0}$, the integral or the FFT. The DMs have shown a good discrimination but the performances are strongly dependent by the considered portion of the signal evolution. Instead, the EV has shown an almost constant classification rate along the time with respect to the temperature modulation being also able to obtain more than $90 \%$ of correct classification rate in the first $5 \mathrm{~s}$. This aspect is of fundamental importance for practical applications since this feature is able to accurately identify and classify different species using few seconds of the signal response independently from the position on the profile of the temperature modulation.

Moreover, further increment in sensing performance is obtained when more Evs, calculated at different time times are taken into account.

Finally conventional sensor arrays using the same type of construction technology have been thoroughly investigated and are widely utilized today in many critical applications. It remains as a standing question, however, how to maximize their performance and simultaneously minimize the time required for it. This is an important question, because sensors composing an array can convey different attributes of the process being monitored, and, when properly conditioned, can complement each other. In this frame, a more complex classification task and the utilization of quality/variety of chemo-sensing technologies using this feature extraction procedure will require more deep investigations.

\section{References}

[1] S. Zaromb and J. R. Stetter, "Theoretical basis for identification and measurement of air contaminants using an array of sensors having partly overlapping selectivities," Sensors and Actuators, vol. 6, no. 4, pp. 225-243, 1984.

[2] K. D. Schierbaum, U. Weimar, and W. Göpel, "Multicomponent gas analysis: an analytical chemistry approach applied to modified $\mathrm{SnO}_{2}$ sensors," Sensors and Actuators B, vol. 2, no. 1, pp. 71-78, 1990.

[3] C. Di Natale, A. D’Amico, F. A. M. Davide, G. Faglia, P. Nelli, and G. Sberveglieri, "Performance evaluation of an $\mathrm{SnO}_{2}$-based sensor array for the quantitative measurement of mixtures of $\mathrm{H}_{2} \mathrm{~S}$ and $\mathrm{NO}_{2}$," Sensors and Actuators B, vol. 20, no. 2-3, pp. 217-224, 1994.

[4] X. Wang, S. Yee, and P. Carey, "An integrated array of multiple thin-film metal oxide sensors for quantification of individual components in organic vapor mixtures," Sensors and Actuators $B$, vol. 13, no. 1-3, pp. 458-461, 1993.

[5] J. W. Gardner, A. Pike, N. F. De Rooij, et al., "Integrated array sensor for detecting organic solvents," Sensors and Actuators B, vol. 26, no. 1-3, pp. 135-139, 1995.

[6] N. Bărsan, R. Ionescu, and A. Vancu, "Calibration curve for $\mathrm{SnO}_{2}$-based gas sensors," Sensors and Actuators B, vol. 19, no. 1-3, pp. 466-469, 1994.

[7] D. M. Wilson, K. Dunman, T. Roppel, and R. Kalim, "Rank extraction in tin-oxide sensor arrays," Sensors and Actuators B, vol. 62, no. 3, pp. 199-210, 2000.

[8] M. Roth, R. Hartinger, R. Faul, and H.-E. Endres, "Drift reduction of organic coated gas-sensors by temperature modulation," Sensors and Actuators B, vol. 36, no. 1-3, pp. 358-362, 1996.

[9] M. Holmberg and T. Artursson, "Drift compensation, standards, calibration methods," in Handbook of Machine Olfaction: Electronic Nose Technology, T. C. Pearce, S. S. Schiffman, H. T. Nagle, and J. W. Gardner, Eds., pp. 325-346, Wiley-VCH, Weinheim, Germany, 2002.

[10] S. W. Moore, J. W. Gardner, E. L. Hines, W. Göpel, and U. Weimar, "A modified multilayer perceptron model for gas mixture analysis," Sensors and Actuators B, vol. 16, no. 1-3, pp. 344-348, 1993.

[11] M. J. Madou and S. R. Morrison, Chemical Sensing with SolidState Devices, Academic Press, Boston, Mass, USA, 1989.

[12] S. Nakata, H. Nakamura, and K. Yoshikawa, "New strategy for the development of a gas sensor based on the dynamic characteristics: principle and preliminary experiment," Sensors and Actuators B, vol. 8, no. 2, pp. 187-189, 1992.

[13] R. E. Cavicchi, J. S. Suehle, K. G. Kreider, M. Gaitan, and P. Chaparala, "Fast temperature programmed sensing for microhotplate gas sensors," IEEE Electron Device Letters, vol. 16, no. 6, pp. 286-288, 1995.

[14] A. Heilig, N. Bârsan, U. Weimar, M. Schweizer-Berberich, J. W. Gardner, and W. Göpel, "Gas identification by modulating temperatures of $\mathrm{SnO}_{2}$-based thick film sensors," Sensors and Actuators B, vol. 43, no. 1-3, pp. 45-51, 1997.

[15] R. E. Cavicchi, J. S. Suehle, K. G. Kreider, M. Gaitan, and P. Chaparala, "Optimized temperature-pulse sequences for the enhancement of chemically specific response patterns from micro-hotplate gas sensors," Sensors and Actuators B, vol. 33, no. $1-3$, pp. $142-146,1996$. 
[16] E. Llobet, R. Ionescu, S. Al-Khalifa, et al., "Multicomponent gas mixture analysis using a single tin oxide sensor and dynamic pattern recognition," IEEE Sensors Journal, vol. 1, no. 3, pp. 207-213, 2001.

[17] E. Llobet, J. Brezmes, R. Ionescu, et al., "Wavelet transform and fuzzy ARTMAP-based pattern recognition for fast gas identification using a micro-hotplate gas sensor," Sensors and Actuators B, vol. 83, no. 1-3, pp. 238-244, 2002.

[18] W. M. Sears, K. Colbow, and F. Consadori, "Algorithms to improve the selectivity of thermally-cycled tin oxide gas sensors," Sensors and Actuators, vol. 19, no. 4, pp. 333-349, 1989.

[19] S. Nakata, S. Akakabe, M. Nakasuji, and K. Yoshikawa, "Gas sensing based on a nonlinear response: discrimination between hydrocarbons and quantification of individual components in a gas mixture," Analytical Chemistry, vol. 68, no. 13, pp. 2067-2072, 1996.

[20] S. Nakata, E. Ozaki, and N. Ojima, "Gas sensing based on the dynamic nonlinear responses of a semiconductor gas sensor: dependence on the range and frequency of a cyclic temperature change," Analytica Chimica Acta, vol. 361, no. 12, pp. 93-100, 1998.

[21] R. Aigner, F. Auerbach, P. Huber, R. Müller, and G. Scheller, "Sinusoidal temperature modulation of the SiPlanar-Pellistor," Sensors and Actuators B, vol. 18, no. 1-3, pp. 143-147, 1994.

[22] A. Vergara, E. Llobet, J. Brezmes, et al., "Optimised temperature modulation of metal oxide micro-hotplate gas sensors through multilevel pseudo random sequences," Sensors and Actuators B, vol. 111-112, pp. 271-280, 2005.

[23] A. Vergara, J. L. Ramírez, and E. Llobet, "Reducing power consumption via a discontinuous operation of temperaturemodulated micro-hotplate gas sensors: application to the logistics chain of fruit," Sensors and Actuators B, vol. 129, no. 1, pp. 311-318, 2008.

[24] F. A. M. Davide, C. D. Natale, A. D'Amico, et al., "Structure identification of non-linear models for $\mathrm{QMB}$ polymer-coated sensors," Sensors and Actuators B, vol. 25, no. 1-3, pp. 830-842, 1995.

[25] G. E. Searle, J. W. Gardner, M. J. Chappell, K. R. Godfrey, and M. J. Chapman, "System identification of electronic nose data from cyanobacteria experiments," IEEE Sensors Journal, vol. 2, no. 3, pp. 218-228, 2002.

[26] D. M. Wilson and S. P. DeWeerth, "Odor discrimination using steady-state and transient characteristics of tin-oxide sensors," Sensors and Actuators B, vol. 28, no. 2, pp. 123-128, 1995.

[27] M. Nakamura, I. Sugimoto, and H. Kuwano, "Application of plasma-polymer-film-coated sensors to gas identification using linear filters," Sensors and Actuators B, vol. 33, no. 1-3, pp. 122-127, 1996.

[28] P. Accettola, M. Balsi, A. D'Amico, C. Di Natale, A. Macagnano, and F. Sortino, "Time-constant extracting filters for fast gas identification in electronic noses," Electronics Letters, vol. 38, no. 11, pp. 510-511, 2002.

[29] J. Samitier, J. M. López-Villegas, S. Marco, et al., "A new method to analyse signal transients in chemical sensors," Sensors and Actuators B, vol. 18, no. 1-3, pp. 308-312, 1994.

[30] F. Takens, "Detecting strange attractors in turbulence," in Dynamical Systems and Turbulence, D. Rand and L. S. Young, Eds., vol. 898 of Lecture Notes in Mathematics, pp. 366-381, Springer, Berlin, Germany, 1980.

[31] E. Martinelli, C. Falconi, A. D'Amico, and C. Di Natale, "Feature extraction of chemical sensors in phase space," Sensors and Actuators B, vol. 95, no. 1-3, pp. 132-139, 2003.
[32] A. Fichera, C. Losenno, and A. Pagano, "Clustering of chaotic dynamics of a lean gas-turbine combustor," Applied Energy, vol. 69, no. 2, pp. 101-117, 2001.

[33] A. Vergara, E. Llobet, E. Martinelli, C. Di Natale, A. D’Amico, and X. Correig, "Feature extraction of metal oxide gas sensors using dynamic moments," Sensors and Actuators B, vol. 122, no. 1, pp. 219-226, 2007.

[34] E. Martinelli, Investigation on alternative approaches to chemical sensors data treatment, Ph.D. thesis, University of Rome Tor Vergata, Rome, Italy, 2003.

[35] A. Vergara, E. Martinelli, E. Llobet, F. Giannini, A. D’Amico, and C. Di Natale, "An alternative global feature extraction of temperature modulated micro-hotplate gas sensors array using an energy vector approach," Sensors and Actuators B, vol. 124, no. 2, pp. 352-359, 2007.

[36] I. Gràcia, J. Santander, C. Cané, M. C Horrillo, I. Sayago, and J. Gutierrez, "Results on the reliability of silicon micromachined structures for semiconductor gas sensors," Sensors and Actuators B, vol. 77, no. 1-2, pp. 409-415, 2001.

[37] M. C. Horrillo, I. Sayago, L. Arés, et al., "Detection of low $\mathrm{NO}_{2}$ concentrations with low power micromachined tin oxide gas sensors," Sensors and Actuators B, vol. 58, no. 1-3, pp. 325-329, 1999.

[38] P. Ivanov, E. Llobet, A. Vergara, et al., "Towards a micro-system for monitoring ethylene in warehouses," Sensors and Actuators B, vol. 111-112, pp. 63-70, 2005.

[39] A. Vergara, E. Llobet, J. Brezmes, et al., "Quantitative gas mixture analysis using temperature-modulated microhotplate gas sensors: selection and validation of the optimal modulating frequencies," Sensors and Actuators B, vol. 123, no. 2, pp. 1002-1016, 2007.

[40] T. Eklöv, P. Mårtensson, and I. Lundström, "Enhanced selectivity of MOSFET gas sensors by systematical analysis of transient parameters," Analytica Chimica Acta, vol. 353, no. 23, pp. 291-300, 1997.

[41] M. Barker and W. Rayens, "Partial least squares for discrimination," Journal of Chemometrics, vol. 17, no. 3, pp. 166-173, 2003.

[42] C. Di Natale, S. Marco, F. Davide, and A. D'Amico, "Sensorarray calibration time reduction by dynamic modelling," Sensors and Actuators B, vol. 25, no. 1-3, pp. 578-583, 1995. 

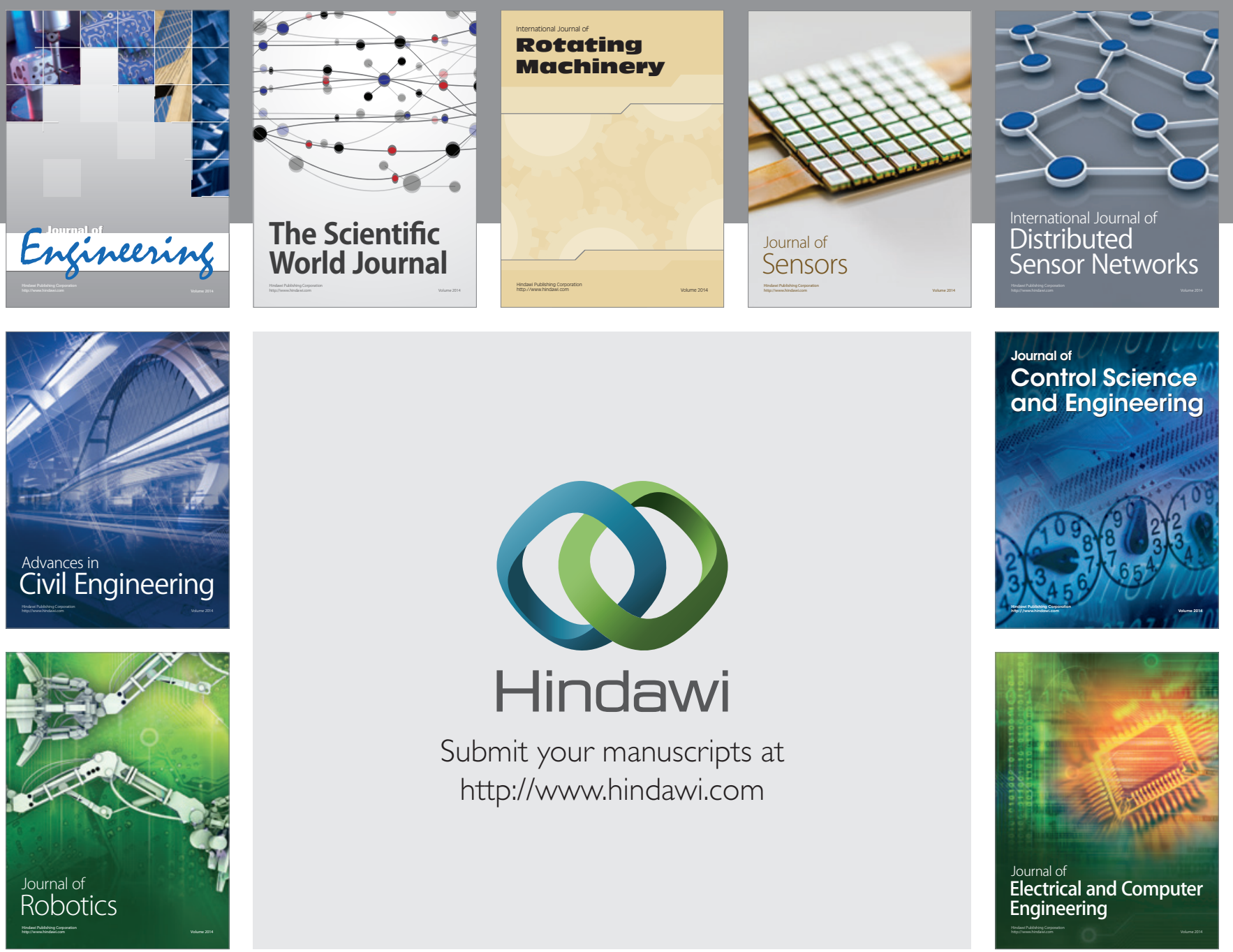

Submit your manuscripts at

http://www.hindawi.com
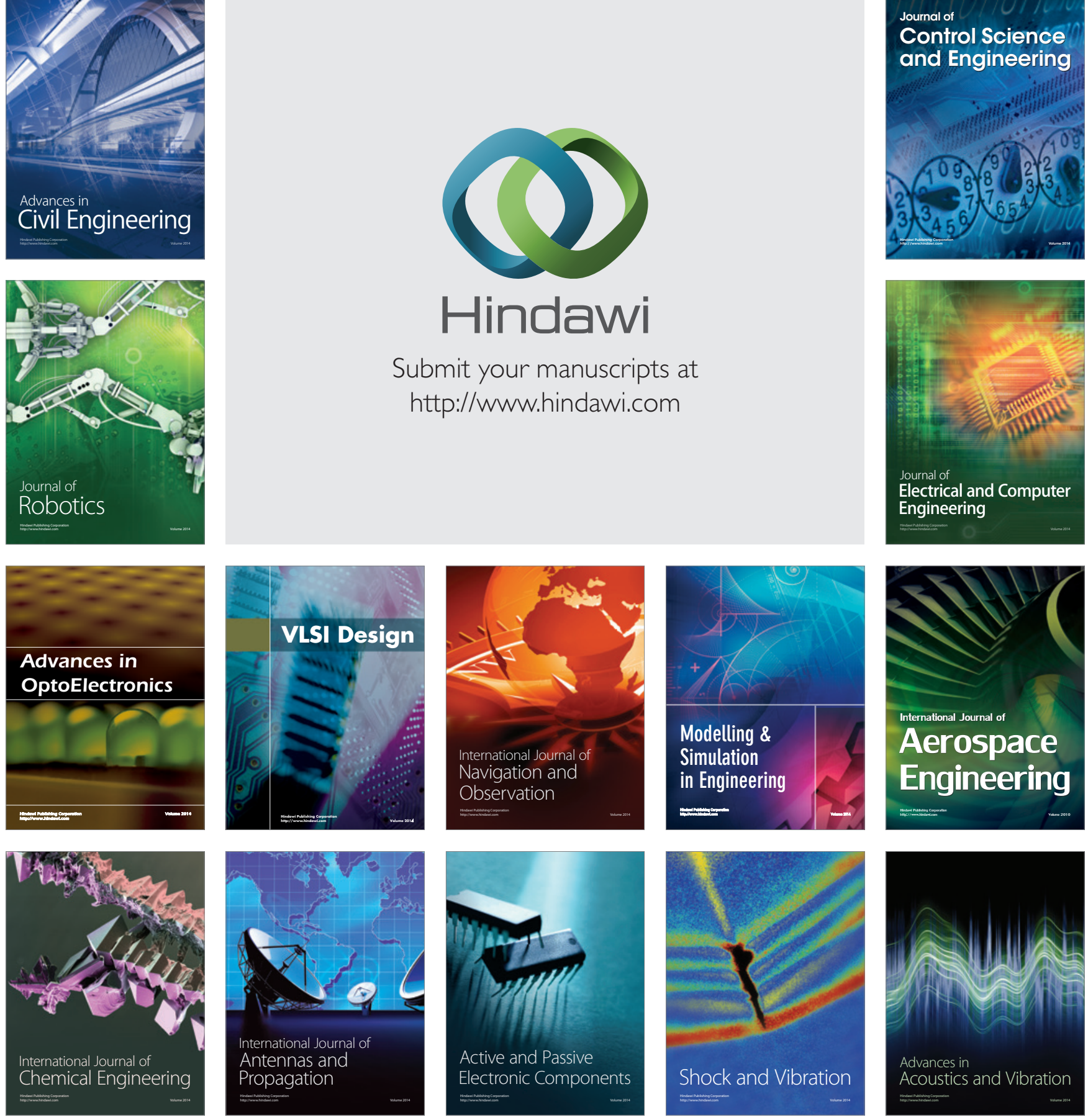